\title{
DYNAMICAL MASS OF GJ 802B: A BROWN DWARF IN A TRIPLE SYSTEM
}

\author{
M. J. IRELAND \\ Division of Geological and Planetary Sciences, California Institute of Technology, Pasadena, CA 91125; mireland@gps.caltech.edu
}

\section{A. KRAuS}

Division of Physics, Mathematics, and Astronomy, California Institute of Technology, Pasadena, CA 91125

\author{
F. Martinache and J. P. Lloyd \\ Cornell University, Ithaca, NY 14853 \\ AND \\ P. G. TUTHILL \\ School of Physics, University of Sydney, NSW 2006, Australia \\ Received 2007 October 9; accepted 2008 January 9
}

\begin{abstract}
We report a dynamical measurement of the mass of the brown dwarf GJ 802B using aperture-masking interferometry and astrometry. In addition, we report the discovery that GJ 802A is itself a close spectroscopic noneclipsing binary with a $19 \mathrm{hr}$ period. We find the mass of GJ $802 \mathrm{~B}$ to be $0.063 \pm 0.005 M_{\odot}$. GJ 802 has kinematics inconsistent with a young star and more consistent with the thick-disk population, implying a system age of $\sim 10$ Gyr. However, model evolutionary tracks for GJ $802 \mathrm{~B}$ predict system ages of $\sim 2 \mathrm{Gyr}$, suggesting that brown dwarf evolutionary models may be underestimating luminosity for old brown dwarfs.
\end{abstract}

Subject headings: stars: low-mass, brown dwarfs

Online material: color figure

\section{INTRODUCTION}

The boundary between stars and brown dwarfs is defined as the mass at which the luminosity of old objects is just dominated by hydrogen burning. This boundary is predicted to occur at a mass between 0.07 and $0.072 M_{\odot}$ at $[\mathrm{Fe} / \mathrm{H}]=0$ and $\sim 0.092 M_{\odot}$ at $[\mathrm{Fe} / \mathrm{H}]=-3$ (Chabrier et al. 2000; Burrows et al. 2001).

However, this boundary, and the theoretical relationships that predict effective temperature and luminosity as a function of mass and age, are largely untested by observations. The observations that are required to test these models are dynamical mass measurements of binary and multiple-star systems, combined with accurate photometry and distance determinations, preferably at known age. Many stars between 0.1 and $0.2 M_{\odot}$ now have accurate dynamical masses (Ségransan et al. 2000), but objects with masses between the hydrogen burning limit and $0.1 M_{\odot}$ so far do not have accurate $(\leqslant 10 \%)$ mass and luminosity determinations.

Dependence on theoretical models has led to controversy about the mass of brown dwarfs, particularly when objects are plausibly near the canonical planetary-mass boundary of $\sim 13 M_{\mathrm{J}}$ (e.g., Luhman et al. 2007). In the few cases in which the dynamical mass of a brown dwarf has been measured (Zapatero Osorio et al. 2004; Stassun et al. 2006), precision is either inadequate to truly constrain models or the model fits depend on unusual assumptions such as noncoeval systems (Stassun et al. 2007).

GJ 802 is a M5 field dwarf system at $\sim 16$ pc that was discovered to have a brown dwarf component through astrometry (Pravdo et al. 2005). The subsequent detection of the companion, GJ 802B, with aperture-masking interferometry (Lloyd et al. 2006) made this an ideal target for dynamical mass determination, due to the system's $\sim 3$ yr period. The high contrast $(\sim 100: 1)$ and small separation ( $\$ 100$ mas) of GJ $802 \mathrm{AB}$ rule out this system for direct imaging observations, but it is ideal for high-contrast interferometric detection.
This paper reports aperture-masking interferometry detections of GJ 802B at six epochs in three colors, enough to make good measurements of the orbit, and the discovery that GJ $802 \mathrm{~A}$ is itself a close spectroscopic M dwarf binary. Section 2 describes the astrometric, interferometric, spectroscopic, and photometric observations that go into the orbit determinations. Section 3 describes the orbit of the close binary GJ 802Aab and $\S 4$ describes the constraint on the dynamical mass of GJ $802 \mathrm{~B}$ from the orbit of GJ $802 \mathrm{AB}$. In $\S 5$ we compare our results to theoretical predictions and $\S 6$ has a summary and discussion.

\section{OBSERVATIONS}

The observations on which this paper is based consist of seeinglimited astrometry, aperture-masking interferometry, infrared and visible spectroscopy, and photometry. The astrometry observations, in addition to parallax and proper motion data, provide the orbit of the GJ 802Aab pair about the GJ 802AB center of mass. The aperture-masking interferometry constrains the wide binary orbit by resolving GJ 802B. The spectroscopy, in the infrared and visible, provides the spectroscopic GJ 802Aab orbit, and the photometry is used to search for eclipses of this inner pair. Each observation set will be discussed in turn.

\subsection{Astrometry Observations}

The astrometry observations in this paper come directly from Pravdo et al. (2005). These observations were part of the Stellar Planet Survey (STEPS) program, with observations made using a custom visible camera mounted at the unfolded Cassegrain focus of the Palomar 200 inch (5 m) Hale Telescope. As the raw astrometry was not published in that paper and has not been made available to us, we extracted the astrometric data from the figures in the paper and then added the motion due to the proper motion and parallax given in the paper's table. These extracted values are 
TABLE 1

Astrometry Observation Summary for GJ 802

\begin{tabular}{|c|c|c|c|}
\hline Date (UT) & $\mathrm{JD}-2,450,000$ & $\begin{array}{c}\Delta \text { R.A. } \\
\text { (mas) }\end{array}$ & $\begin{array}{c}\Delta \text { decl. } \\
\text { (mas) }\end{array}$ \\
\hline 1998 Jul 6 ..................... & 1000.75 & 0.00 & 0.00 \\
\hline 1999 Jul $20 \ldots \ldots \ldots \ldots \ldots . .$. & 1379.75 & 901.50 & 1806.99 \\
\hline 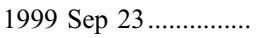 & 1444.75 & 1002.89 & 2092.99 \\
\hline 2000 Jul 13 ................... & 1738.75 & 1780.05 & 3510.93 \\
\hline 2001 Jul $2 \ldots \ldots \ldots \ldots \ldots \ldots$ & 2092.75 & 2631.23 & 5150.19 \\
\hline 2002 Jul 7 ................... & 2462.75 & 3513.06 & 6908.15 \\
\hline 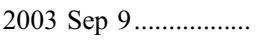 & 2891.75 & 4496.65 & 8932.57 \\
\hline 2004 Jul 12 ................. & 3198.75 & 5276.92 & $10,367.24$ \\
\hline 2004 Sep $15 \ldots \ldots \ldots \ldots \ldots$ & 3263.75 & 5371.75 & $10,654.40$ \\
\hline
\end{tabular}

presented in Table 1 and include all epochs for which there are at least two measurements given in Pravdo et al. (2005). We give all of these epochs equal weight. The error estimated from the scatter within individual epochs is 1.6 mas, although we assume errors of 1.7 mas, to give a final fitted $\chi^{2}$ of 1.0 (see $\left.\S 4\right)$.

\subsection{Aperture-masking Interferometry}

The technique of nonredundant aperture masking has been well established as a means of achieving the full diffraction limit of a single telescope (e.g., Michelson 1920; Baldwin et al. 1986; Tuthill et al. 2000). It involves placing a mask with an array of holes of nonredundant spacing in the pupil plane of a telescope, and analyzing the recorded images as interference fringes on a number of discrete baselines. Recently, our group has used this technique using cameras behind adaptive optics (AO) at the Palomar 200 inch and Keck telescopes to increase the magnitude limit of the technique beyond that achievable in a seeing-limited speckle mode. The reason for the technique's success over direct AO imaging is that the calibration is independent of structure of the wave front over scales larger than a single subaperture, while it still preserves the angular resolution of the full aperture.

Aperture-masking observations of GJ 802 were made using both the PHARO camera of the Palomar 200 inch telescope (Hayward et al. 2001) and the NIRC2 camera of the Keck II telescope. The Palomar aperture-masking mode of the PHARO camera is described in Lloyd et al. (2006). The NIRC2 aperture-masking mode is similar, although the masks are placed in the filter wheels of the NIRC2 camera rather than in a designated pupil wheel, as is the case with PHARO. The masks create fringe patterns in the image plane of each detector. Our primary observable, closure phase, is extracted from these fringe patterns and models are fit to the closure phases. A summary of all aperture-masking observations, along with the detected binary properties, is given in Table 2 .
For the NIRC2 and PHARO aperture-masking experiments, both 9- and 18-hole masks are available. The 9-hole mask results in 36 simultaneous baselines and 28 independent closure phases. The 18-hole mask results in 153 simultaneous baselines and 136 independent closure phases, but has half the throughput of the 9-hole mask and spreads the light over 4 times as many pixels as the 9-hole mask.

All observations were done with a 9-hole mask in $H$ and $K$ bands, except the 2007 June $J_{\text {cont }}$ observation for which an 18-hole mask was used. In the absence of sky rotation, the field of view of the aperture-masking experiment is given by $\lambda / 2 B_{S}$, where $B_{S}$ is the shortest baseline in the mask. Outside this field of view, the position angle and separation become ambiguous without multiple exposures at different sky rotations. Although the 9-hole mask was well suited to $H$ and $K$ observations at both Keck and Palomar, the $\sim 73$ mas separation of GJ 802B would have been outside the nominal 9-hole mask field of view in $J$ band.

In a similar manner, the aperture-masking inner working angle is given by $\lambda / 2 B_{L}$, where $B_{L}$ is the longest baseline (near to the full aperture size). At this separation, the maximum closure phase signal is equal to the contrast ratio of the binary (i.e., $0.01 \mathrm{rad}$ or $0.6^{\circ}$ for a $100: 1$ binary). In the case of the PHARO observations of 2004 June and 2006 October, these nominal inner detection limits are 43 and 57 mas, respectively. Near this inner edge, the contrast ratio and separation for model-fitting are degenerate. Despite their large errors on separation, these detections are therefore reliable. We explicitly list the correlations between separation and contrast in Table 2.

We have made several improvements to the analysis pipeline and observing procedure since the original detection published in Lloyd et al. (2006). The most important improvement has been in the observations themselves: we have generally been much more careful in using calibrators of similar brightness, colors, and position in the sky. This procedure was not used carefully for the discovery epoch of 2004 September, and we find that the astrometry depends on which of several unsuitable calibrators are used in the analysis. Therefore, we choose not to use this epoch in our analysis here, and it is not presented in Table 2.

We have windowed the data prior to Fourier-transforming with a tighter function than was used in previous publications to minimize residual chip-based effects (e.g., of bad pixels). The window size is of the form $\exp \left(-r^{4}\right)$ with a FWHM of $1.6 \lambda / D_{H}$, where $D_{H}$ is our aperture-mask hole diameter projected onto the primary mirror. This tighter window also has the effect of spatially filtering the interferometric data. The raw frames from the PHARO camera are taken in a mode in which all (nondestructive) reads are saved. This allows us to split the data in postprocessing into a variety of integration times, where smaller integration times have less

TABLE 2

Aperture-masking Observation Summary for GJ 802

\begin{tabular}{|c|c|c|c|c|c|c|c|}
\hline Date (UT) & $\mathrm{JD}-2,450,000$ & Instrument & Filter & $\begin{array}{l}\text { Separation } \\
\text { (mas) }\end{array}$ & $\begin{array}{c}\text { Position Angle } \\
\text { (deg) }\end{array}$ & $\begin{array}{l}\text { Contrast } \\
(\mathrm{B} / \mathrm{A})\end{array}$ & $\begin{array}{c}\text { Separation-Contrast } \\
\text { Correlation }\end{array}$ \\
\hline 2004 Jun 6 .............. & $53,163.0$ & PHARO & $\mathrm{H}$ & $55.1 \pm 21.4$ & $16.4 \pm 6.8$ & $0.013 \pm 0.006$ & 0.87 \\
\hline 2006 Jun $23 \ldots \ldots \ldots \ldots$ & $53,910.0$ & NIRC2 & $\mathrm{Ks}$ & $85.8 \pm 3.5$ & $200.9 \pm 2.2$ & $0.015 \pm 0.003$ & 0.09 \\
\hline 2006 Oct $10 \ldots \ldots \ldots \ldots$ & $54,018.7$ & PHARO & $\mathrm{H}$ & $94.6 \pm 9.7$ & $207.6 \pm 7.4$ & $0.009 \pm 0.003$ & -0.13 \\
\hline 2006 Oct $10 \ldots \ldots \ldots \ldots$ & $54,018.7$ & PHARO & Ks & $81.1 \pm 24.5$ & $196.7 \pm 4.9$ & $0.011 \pm 0.003$ & 0.75 \\
\hline 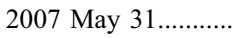 & $54,252.0$ & PHARO & $\mathrm{CH} 4 \mathrm{~S}$ & $77.4 \pm 10.3$ & $24.1 \pm 5.2$ & $0.009 \pm 0.002$ & -0.26 \\
\hline 2007 Jun 5 .............. & $54,257.0$ & NIRC2 & Jcont & $73.3 \pm 2.9$ & $21.5 \pm 2.1$ & $0.008 \pm 0.002$ & 0.04 \\
\hline 2007 Jun $6 \ldots \ldots \ldots . . . .$. & $54,258.0$ & $\mathrm{NIRC} 2$ & $\mathrm{Kp}$ & $72.6 \pm 1.7$ & $19.2 \pm 1.2$ & $0.010 \pm 0.001$ & 0.01 \\
\hline 2007 Jul 31 ............. & $54,312.9$ & PHARO & $\mathrm{H}$ & $86.2 \pm 5.8$ & $24.0 \pm 3.5$ & $0.010 \pm 0.002$ & -0.40 \\
\hline 2007 Aug $29 . . . \ldots \ldots . . .$. & $54,341.8$ & PHARO & $\mathrm{H}$ & $85.4 \pm 6.0$ & $20.2 \pm 3.2$ & $0.011 \pm 0.002$ & -0.44 \\
\hline
\end{tabular}


atmospheric and AO system noise but more readout noise. We now carefully choose an optimal number of subreads to analyze data of a given brightness: for GJ 802 we split the data into subframes spaced by two reads, which have integration times of $862 \mathrm{~ms}$ each.

\subsection{Infrared Spectroscopy}

The NIRC2 camera at the Keck II telescope was used on UT 2006 Aug 10 in a spectroscopic mode for the purpose of attempting to detect the spectrum of GJ 802B directly. The observations were made in the $H$ band with a bandpass from 1.52 to $1.62 \mu \mathrm{m}$. We used a grism with a resolving power of 17,580 pixel $^{-1}$ and a 4 pixel wide slit, giving a spectral resolution of $\sim 4000$. These observations did not succeed in their primary purpose, mainly because poor seeing forced us to use $H$ instead of $J$ band, where the spectral differences between a mid-L and mid-M dwarf were not large enough to give a clearly detectable GJ 802B signal in the data.

It was noticed, however, that lines in the spectra of GJ 802 were double. To calibrate the spectra, we first used Xe and Ar lamps to calibrate the wavelength scale, which was fitted with a secondorder polynomial. We calculated the model atmospheric and grism transmission by taking spectra of the F8V star HD 136118 and dividing by a template spectrum of HR 4375 (a G0 V star) from Meyer et al. (1998). In reducing these spectra to their two components, GJ 777B was used as the spectral-type standard and the radial velocity standard, as its wide companion GJ 777A has a precise radial velocity of $-45.350 \pm 0.004 \mathrm{~km} \mathrm{~s}^{-1}$ (Naef et al. 2003 ) and should be within $1 \mathrm{~km} \mathrm{~s}^{-1}$ from GJ 777B's radial velocity due to potential orbital motion. As a check of our calibration procedure, we used Xe and Ar lamp calibration of the NIRC2 grism wavelength scale to measure the radial velocity of GJ 777B based on two $\mathrm{Al} \mathrm{I}$ and one $\mathrm{Ca}$ I lines to be $-41 \pm 5 \mathrm{~km} \mathrm{~s}^{-1}$, consistent with GJ 777A's radial velocity.

Least-square fits were made to the continuum-subtracted spectra of GJ 802, based on a model made up of the sum of two shifted GJ 777B spectra. We chose this technique rather than a crosscorrelation because the two spectra were not separated well enough to give clearly separate peaks in the cross-correlation. The errors in the velocity difference come straight from the least-squares fitting process, where the uncertainty in the GJ 802 spectrum was set to $1.3 \%$ of the mean flux, so that the model fit had a reduced $\chi^{2}$ of 1 . An uncertainty on the velocity difference based on deviations from a linear fit to the measured velocities would be $2.5 \mathrm{~km} \mathrm{~s}^{-1}$. The results of this fitting process are given in Table 3 . The magnitude difference in $H$ band derived from the fit is zero within errors, which suggests that the components have nearly equal masses.

It is difficult to estimate the uncertainties on the absolute velocity calibration (which was not the primary purpose of our observations), so we assign an error of $5 \mathrm{~km} \mathrm{~s}^{-1}$ based on the absolute calibration of GJ 777B's radial velocity against the Xe and Ar lamps. There is good reason to expect that the absolute calibration of these observations is significantly worse than the relative calibration between the $\mathrm{Aa}$ and $\mathrm{Ab}$ spectra, because the pointspread function delivered by the AO system was not guaranteed to be well centered on the slit. However, as both stars had the same point-spread function, with their maximum possible spatial separation of 0.7 mas corresponding to $\sim 1 \mathrm{~km} \mathrm{~s}^{-1}$, the relative calibration between Aa and Ab spectra should be good compared with the $2 \mathrm{~km} \mathrm{~s}^{-1}$ statistical error in the velocity difference.

\subsection{High-Resolution Optical Spectroscopy}

We obtained multiepoch spectra of GJ 802 using the East Arm Echelle (EAE) on the Palomar 200 inch telescope. The EAE is a high-resolution spectrograph, capable of achieving spectral reso-
TABLE 3

INFraRed Radial Velocities for GJ 802Aab

\begin{tabular}{lrccr}
\hline \hline $\mathrm{JD}-2,450,000$ & \multicolumn{1}{c}{$\mathrm{RV}_{\mathrm{Ab}}$} & \multicolumn{1}{c}{$\mathrm{RV}_{\mathrm{Aa}}$} & $\Delta \mathrm{RV}$ & \multicolumn{1}{c}{$H_{\mathrm{Aa}}-H_{\mathrm{Ab}}{ }^{\mathrm{a}}$} \\
\hline $3,956.9219 \ldots \ldots$. & $-6.9 \pm 5.0$ & $-93.5 \pm 5.0$ & $-86.6 \pm 2.0$ & $0.15 \pm 0.06$ \\
$3,956.9336 \ldots \ldots$. & $-13.2 \pm 5.0$ & $-86.1 \pm 5.0$ & $-72.9 \pm 2.0$ & $0.19 \pm 0.06$ \\
$3,956.9375 \ldots \ldots$. & $-12.9 \pm 5.0$ & $-83.7 \pm 5.0$ & $-70.9 \pm 2.0$ & $0.24 \pm 0.06$ \\
$3,956.9453 \ldots \ldots$. & $-16.3 \pm 5.0$ & $-83.6 \pm 5.0$ & $-67.3 \pm 2.0$ & $-0.03 \pm 0.07$
\end{tabular}

a Difference in apparent $H$-band magnitudes as derived from the fit of two shifted GJ 777B spectra. See text.

lution of $R \sim 30,000$. Most of our observations span a wavelength range of 4000-10000 A, but the observations taken in 2006 December (before official commissioning) span a significantly bluer range (3500-8000 $\AA$ ). Most of our spectra were binned in the spectral direction in order to reduce read noise, so the effective resolution for our observations was $R \sim 20,000$. Since GJ 802 is very red, none of our spectra have any usable signal shortward of $\sim 5500 \AA$.

We observed GJ 802 at four epochs on 2006 December 15 and at six more epochs spanning 2007 May 6-8. We also observed the late-type stars GJ 51 (in December) and GJ 581, GJ 686, and GJ 699 (in May) as spectral-type standards. All observations of GJ 802 used integration times of 300-900 s, while our standardstar observations used a wider range of integration times, since some were significantly brighter.

We bias-subtracted, flat-fielded, and extracted our spectra using standard IRAF tasks. Wavelength calibration was achieved with respect to a thorium-argon lamp that was observed at the beginning of each night; preliminary tests suggest that telescope flexure results in wavelength calibration variations of no more than $\sim 0.015 \AA\left(\sim 0.5 \mathrm{~km} \mathrm{~s}^{-1}\right)$ in the vicinity of the $\mathrm{H} \alpha$ emission line at $6563 \AA$. In Figure 1 we show a representative segments of several spectra for GJ 802 around the $\mathrm{H} \alpha$ wavelength range. These spectra demonstrate the double-lined nature of GJ 802; this plot also shows that the $\mathrm{H} \alpha$ emission-line strengths are not constant, but vary on a timescale of days.

All of our science spectra exhibit $\mathrm{H} \alpha$ emission, so we have directly determined the component radial velocities from the line centroids. For epochs in which the lines were not clearly resolved, we fit the spectra with a pair of Gaussian emission lines with the same FWHM as the resolved measurements. We list all of these radial velocities, including heliocentric corrections, in Table 4; we also list our observed radial velocity standards in Table 5 . The mean radial velocity of the standards was $3.0 \mathrm{~km} \mathrm{~s}^{-1}$ higher than the radial velocities derived from the thorium-argon lamp calibration, with a dispersion of $1.7 \mathrm{~km} \mathrm{~s}^{-1}$ (standard error on the mean $0.5 \mathrm{~km} \mathrm{~s}^{-1}$ ). We have subtracted this offset from all radial velocities reported in Tables 5 and 4.

We did not use any cross-correlation techniques to determine overall RV fits because we are still attempting to characterize the wavelength and flux calibrations of the instrument, including limits on potential variability; given the long time baseline of our data set, any improvement in the precision would yield only very minor improvements in the orbital ephemerides.

\subsection{Photometry}

Given that the GJ 802Aab binary had such a short period, and preliminary analysis of the orbit suggested that inclination was possibly high, we used the Palomar 60 inch $(1.5 \mathrm{~m})$ robotic telescope to search for eclipses. The Palomar 60 inch telescope has a single instrument, a CCD imager with an $11^{\prime} \times 11^{\prime}$ field of view and a selection of broad- and narrowband filters. We took series 

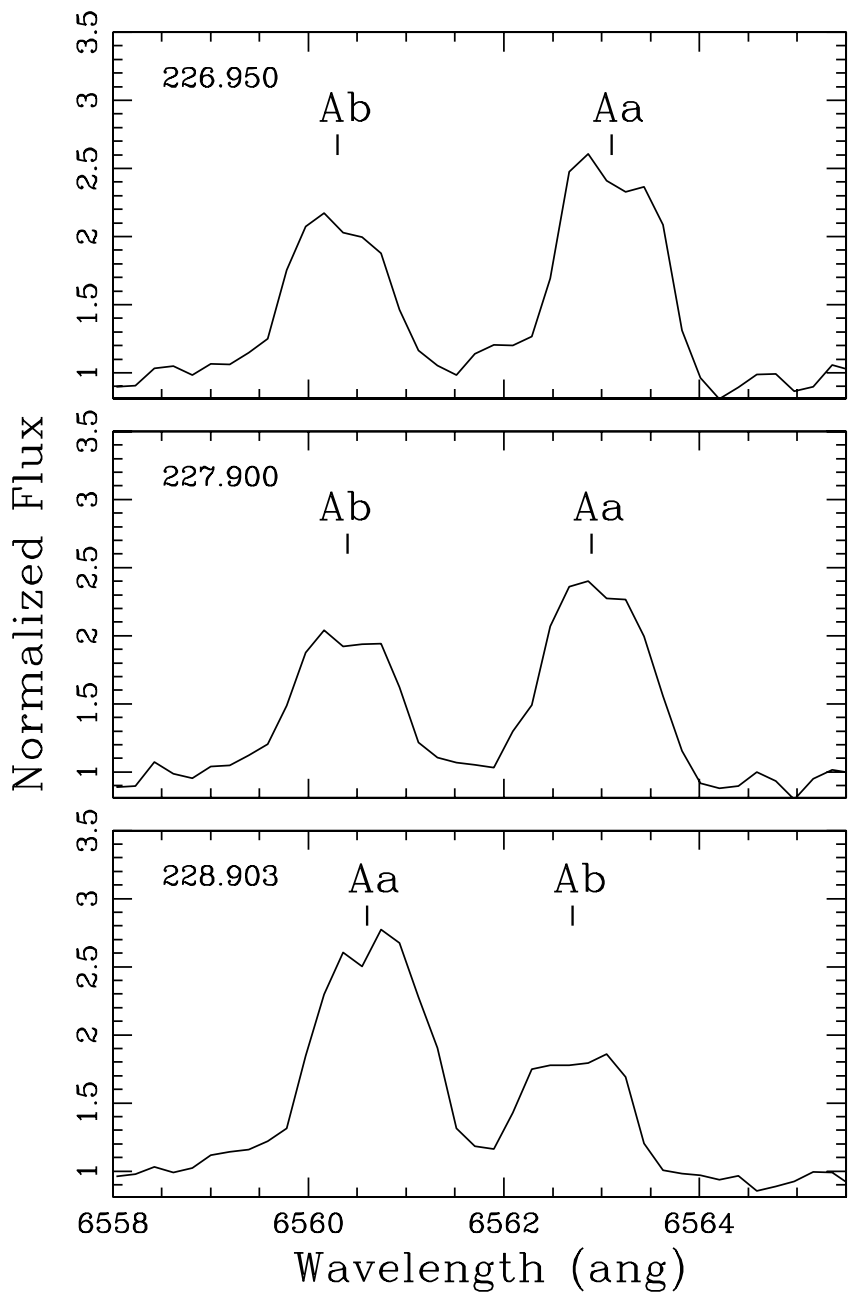

FIG. 1.-High-resolution spectra for GJ 802 centered on the $\mathrm{H} \alpha$ emission line. Both components show resolved $\mathrm{H} \alpha$ emission, but the $\mathrm{H} \alpha$ emission-line strengths appear to be variable on a timescale of days.

of images in $g, i$, and $\mathrm{H} \alpha$ filters around the predicted times of eclipses based on the radial velocity orbit (when the velocity difference was zero). The $\mathrm{H} \alpha$ filter was used to search for an eclipse of the chromospheric emission.

The observations in $g$ and $i$ filters spanned 30 minutes on either side of a predicted eclipse at Julian day 2,454,311.889, and the observations in $\mathrm{H} \alpha$ spanned 30 minutes on either side of a predicted eclipse at Julian day 2,454,315.870. These eclipse times were based on a preliminary orbital fit (see $\S 3$ ). As the radial velocity amplitudes of $\mathrm{Aa}$ and $\mathrm{Ab}$ were roughly equal (see the ve-

TABLE 4

High-Resolution Spectroscopy of GJ 802

\begin{tabular}{crrr}
\hline \hline $\mathrm{JD}-2,450,000$ & \multicolumn{1}{c}{$\mathrm{RV}_{\mathrm{Ab}}$} & \multicolumn{1}{c}{$\mathrm{RV}_{\mathrm{Aa}}$} & \multicolumn{1}{c}{$\Delta \mathrm{RV}$} \\
\hline $4084.57847 \ldots \ldots \ldots \ldots$. & $-107.7 \pm 5.0$ & $10.2 \pm 5.0$ & $-117.9 \pm 2.8$ \\
$4084.59097 \ldots \ldots \ldots \ldots$. & $-111.8 \pm 5.0$ & $15.7 \pm 5.0$ & $-127.5 \pm 2.8$ \\
$4084.60347 \ldots \ldots \ldots \ldots$. & $-118.2 \pm 5.0$ & $18.0 \pm 5.0$ & $-136.2 \pm 2.8$ \\
$4084.70625 \ldots \ldots \ldots \ldots$. & $-116.9 \pm 6.0$ & $19.4 \pm 6.0$ & $-136.3 \pm 4.2$ \\
$4084.71389 \ldots \ldots \ldots \ldots$. & $-118.7 \pm 6.0$ & $18.5 \pm 6.0$ & $-137.2 \pm 4.2$ \\
$4084.72014 \ldots \ldots \ldots \ldots$. & $-113.2 \pm 7.0$ & $16.6 \pm 7.0$ & $-129.8 \pm 7.1$ \\
$4226.94971 \ldots \ldots \ldots \ldots$. & $-102.3 \pm 2.0$ & $23.2 \pm 2.0$ & $-125.5 \pm 2.8$ \\
$4227.90039 \ldots \ldots \ldots \ldots$. & $-99.3 \pm 2.0$ & $17.9 \pm 2.0$ & $-117.2 \pm 2.8$ \\
$4228.84473 \ldots \ldots \ldots \ldots$. & $-21.4 \pm 2.0$ & $-59.8 \pm 2.0$ & $38.4 \pm 2.8$ \\
$4228.90332 \ldots \ldots \ldots \ldots$. & $9.7 \pm 2.0$ & $-86.7 \pm 2.0$ & $96.4 \pm 2.8$ \\
\hline
\end{tabular}

TABLE 5

High-Resolution Spectroscopy of Standards

\begin{tabular}{|c|c|c|c|}
\hline Star & $\mathrm{JD}-2,450,000$ & RV & Literature RV \\
\hline GJ $51 \ldots \ldots \ldots \ldots \ldots$ & 4084.66134 & $-10.9 \pm 3$ & $-7.7^{\mathrm{a}}$ \\
\hline GJ $393 \ldots \ldots \ldots \ldots \ldots . . . . . . .$. & 4226.70127 & $+4.6 \pm 3$ & $+8.3^{\mathrm{b}}$ \\
\hline GJ 476 .................... & 4226.75556 & $+29.6 \pm 3$ & $+31.6^{\mathrm{a}}$ \\
\hline GJ $686 \ldots \ldots \ldots \ldots \ldots$ & 4226.83443 & $-11.4 \pm 3$ & $-9.5^{\mathrm{b}}$ \\
\hline 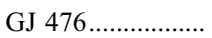 & 4227.73785 & $+30.6 \pm 3$ & $+31.6^{\mathrm{a}}$ \\
\hline 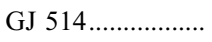 & 4227.74175 & $+11.2 \pm 3$ & $14.6^{\mathrm{b}}$ \\
\hline GJ $526 \ldots \ldots \ldots \ldots \ldots$ & 4227.74530 & $+13.0 \pm 3$ & $16.0^{\mathrm{a}}$ \\
\hline GJ $581 \ldots \ldots \ldots \ldots \ldots$ & 4227.74907 & $-13.8 \pm 3$ & $-9.4^{\mathrm{b}}$ \\
\hline GJ $393 \ldots \ldots \ldots \ldots \ldots$ & 4228.63097 & $+4.9 \pm 3$ & $+8.3^{\mathrm{b}}$ \\
\hline GJ $387.2 \ldots \ldots \ldots \ldots \ldots$ & 4228.64279 & $-16.8 \pm 3$ & $-16.5^{\mathrm{a}}$ \\
\hline GJ $699 \ldots \ldots \ldots \ldots \ldots . . . . . . .$. & 4228.82439 & $-116.9 \pm 3$ & $-110.5^{b}$ \\
\hline
\end{tabular}

${ }^{\text {a }}$ Gizis et al. (2002).

b Nidever et al. (2002).

locities in Table 4), we can assume that the masses are roughly equal and that the primary and secondary eclipses would have roughly equal depths.

We computed aperture photometry for GJ 802 on the calibrated images, using the median photometric variation of 10 nearby field stars to compute the sky transmission in each frame. No eclipses were found in any filters. For the $g$ and $i$ filters, the rms photometry scatter was 0.01 and $0.003 \mathrm{mag}$, respectively, with sampling at 1.5 minute intervals. For the $\mathrm{H} \alpha$ filter, the scatter was $0.01 \mathrm{mag}$, and the sampling at 1 minute intervals. The excess in our filter due to the $\mathrm{H} \alpha$ emission was $22.7 \%$ of the continuum, calculated by comparison with photometry from an off-line narrowband filter and consistent with our spectroscopic observations. From these data we can place $2 \sigma$ upper limits for a continuum eclipse depth of 0.005 mag and for a $\mathrm{H} \alpha$ eclipse depth of $0.01 \mathrm{mag}$.

\section{ORBIT OF THE CLOSE PAIR GJ 802A}

We chose to fit the radial velocities of GJ 802Aab with a circular orbit only, because the $19 \mathrm{hr}$ period of the system is 10 times shorter than the canonical cutoff of 7 days for tidal circularization in low-mass main-sequence stars (Zahn \& Bouchet 1989). Figure 2 shows the best-fit equal-mass circular orbit with the measured radial velocities, and Figure 3 shows the best-fit circular orbit with the measured radial velocity differences. We made this fit by first examining by eye the 2007 May radial velocities,

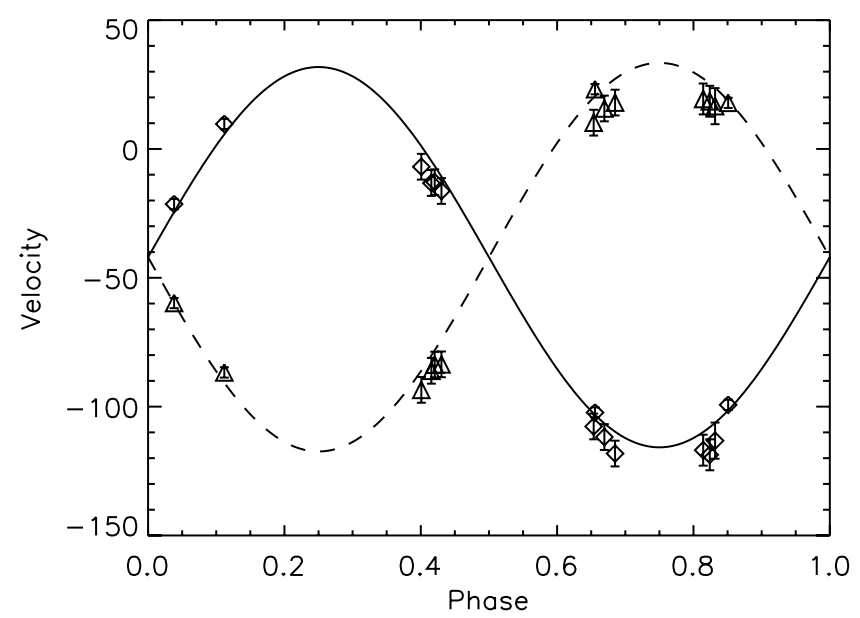

FIG. 2.-Best-fit orbit for GJ 802Aab, showing the Aa velocities (solid line, diamonds) and the $\mathrm{Ab}$ velocities (dashed line, triangles). 


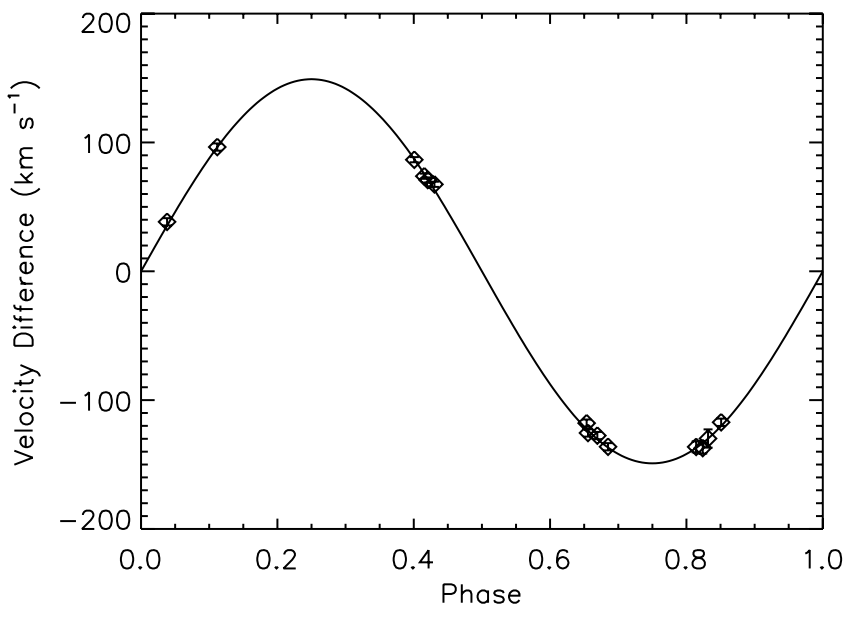

FIG. 3.-Best-fit orbit for GJ 802Aab, showing the difference in velocities between the two components.

concluding that the orbital semiamplitude was between 135 and $155 \mathrm{~km} \mathrm{~s}^{-1}$, with a period of approximately 0.8 days. We then fit to the absolute value of the velocity difference by using a grid search. We searched the range of 0.7 to 0.9 days with $10^{-5}$ days spacing for period, the full range of epoch (modulo half a period) with $10^{-3}$ days spacing, and total velocity amplitudes of the two components $K 1+K 2$, between 135 and $155 \mathrm{~km} \mathrm{~s}^{-1}$, with $4 \mathrm{~km} \mathrm{~s}^{-1}$ spacing. The reduced $\chi^{2}$ for the final fit is 0.70 with 11 degrees of freedom. No other (aliased) minima have reduced $\chi^{2}$ values less than 6 . We assigned signs to the radial velocity differences only after finding this fit, as GJ 802Aab is so close to equal brightness that it was difficult to tell which spectrum was GJ 802Aa and which was GJ 802Ab.

Best-fit orbital parameters are given in Table 6. It is not clear from these observations which component is the more massive, as the mass ratio $M_{\mathrm{Ab}} / M_{\mathrm{Aa}}(=K 1 / K 2)$ is $0.98 \pm 0.03$. This ambiguity is seen in Figure 2. The mass ratio and center of mass radial velocity are constrained best by the 2007 May observations, which are best calibrated and in which the two spectra swap their positions, and the absolute calibration of the radial velocities is most certain. These data have significantly smaller errors in Figure 2 and are at phase $\sim 0.03,0.1,0.65$, and 0.85 . Including a $\sim 3 \mathrm{~km} \mathrm{~s}^{-1}$ uncertainty for the orbital motion of GJ 802Aab with respect to the GJ $802 \mathrm{AB}$ center of mass, we obtain a radial velocity for the system of $-42 \pm 4 \mathrm{~km} \mathrm{~s}^{-1}$.

The inclination limit in Table 6 comes from the following analysis of both the lack of eclipses and a model-dependent mass estimate for the system. Based on a stellar radius of $0.16 R_{\odot}$, predicted by the models of Baraffe et al. (1998) for components of mass $\sim 0.14 M_{\odot}$ [applicable to an edge-on system with equal-mass components and $\left(M_{\mathrm{Aa}}+M_{\mathrm{Ab}}\right) \sin ^{3} i$ from Table 6], the eclipse would have lasted a maximum of 50 minutes. For a grazing eclipse with $5.8 \%$ of one star eclipsed, the eclipse would have lasted 25 minutes, well within our 60 minute observing window ( $\$ 2.5)$. A grazing eclipse occurs at a projected separation of twice the stellar radius, or $\sim 0.32 R_{\odot}$. As no eclipse occulting even $1 \%$ of the surface of one of the stars was found in any filter $(\S 2.5)$, the inclination of GJ $802 \mathrm{Aab}$ is limited to $<83^{\circ}$. Given that $\left(M_{\mathrm{Aa}}+\right.$ $\left.M_{\mathrm{Ab}}\right) \sin ^{3} i=0.273 \pm 0.008$, this in turn limits the total mass of GJ 802 A to $>0.279 \pm 0.008$.

The models of Baraffe et al. (1998) have been verified to predict the $K$-band mass-luminosity relationship for field dwarfs correctly to within $\sim 5 \%$ in mass for dwarfs of mass greater than $0.1 M_{\odot}$ (Delfosse et al. 2004). For stars of mass $\sim 0.11-0.18 M_{\odot}$,
TABLE 6

Radial Velocity Solution for GJ 802Aab

\begin{tabular}{|c|c|}
\hline Parameter & Value \\
\hline Epoch (HJD).. & $2,454,140.530 \pm 0.001$ \\
\hline Period (days) .................. & $0.795340 \pm 0.000003$ \\
\hline 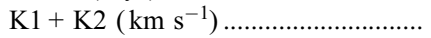 & $149.1 \pm 1.5$ \\
\hline $\mathrm{K} 1\left(\mathrm{~km} \mathrm{~s}^{-1}\right)$ & $73.8 \pm 1.4$ \\
\hline $\mathrm{K} 2\left(\mathrm{~km} \mathrm{~s}^{-1}\right) .$. & $75.4 \pm 1.4$ \\
\hline$\left(M_{\mathrm{Aa}}+M_{\mathrm{Ab}}\right) \sin ^{3} i\left(M_{\odot}\right) \ldots \ldots \ldots \ldots \ldots \ldots$ & $0.273 \pm 0.008$ \\
\hline 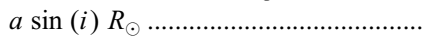 & $2.343 \pm 0.024$ \\
\hline Inclination $(\mathrm{deg}) \ldots$ & $77<i<83$ \\
\hline
\end{tabular}

this relationship is independent of age at the $1 \%$ level for ages between 0.5 and $10 \mathrm{Gyr}$. This age range is applicable to the likely GJ 802 age of $\gtrsim 6$ Gyr, based on its activity (Pravdo et al. 2005) and kinematics $(\S 5)$. This relationship is also relatively steep: at a mass of $0.13 M_{\odot}$, a $6 \%$ error in $K$-band flux from the distance of Pravdo et al. (2005) translates to only a $2.5 \%$ error in mass. Therefore, the uncertainty in the mass is likely dominated by the $K$-band mass-luminosity relationship (at least until the models are tested at higher precision), and we assign a standard deviation of $5 \%$ to the model-predicted mass. These models predict masses for GJ 802Aa and GJ 802Ab of $0.134 M_{\odot}$, using a parallax of 63 mas (Pravdo et al. 2005) and 2MASS photometry. In turn, this places a maximum-mass constraint on GJ 802Aab at $\sim 2 \sigma$ of $0.295 \mathrm{M}_{\odot}$, limiting the inclination of GJ 802Aab to between $77^{\circ}$ and $83^{\circ}$. Note that the $K$-band luminosity-mass relationship is little affected by metallicity, and would change by $<1 \%$ if we were to use the models of Montalbán et al. (2000) at $[\mathrm{Fe} / \mathrm{H}]=-1$.

\section{DYNAMICAL MASS OF GJ 802B}

The full solution for the GJ 802AB orbit requires 16 parameters: 5 for parallax and proper motion, 7 for the orbital solution of $\mathrm{B}$ with respect to Aab, 1 for the photocenter semimajor axis, and 3 for the contrast of B with respect to Aab in $J, H$, and $K$ bands. The reason contrast had to be added in to the orbital solution was that many of the parameters in Table 2 had strong degeneracies. To fully explore this large parameter space around the best-fit solution, we used a Markov chain Monte Carlo technique (e.g., Brémaud 1999), a method that has often been used in astronomy for cosmological parameter estimation (e.g., Knox et al. 2001). Key advantages of this technique are the ability to easily include the covariance matrix of the data (in our case the aperture-masking fits) and the ability to easily calculate the posterior probability function of derived parameters, such as the mass of GJ 802B.

The probability that a particular set of parameters is contained in the final chain is proportional to the likelihood function, which is proportional to $\exp \left(\chi^{2} / 2\right)$. Due to the correlations between derived parameters from aperture-masking, $\chi^{2}$ is not just the sum of normalized deviates, but makes use of the covariance matrix of the data:

$$
\chi^{2}=[\boldsymbol{m}(\boldsymbol{\theta})-\boldsymbol{d}]^{t} C^{-1}[\boldsymbol{m}(\boldsymbol{\theta})-\boldsymbol{d}] .
$$

Here $\boldsymbol{d}$ is the vector of data values, $\boldsymbol{m}$ is the model for these data based on parameters $\boldsymbol{\theta}, C$ is the data covariance matrix, and the superscript $t$ represents a transpose. Covariances between different epochs were assumed to be zero: only the derived separation, position angle, and contrast for a single epoch of aperture-masking data had nonzero covariances.

We used a Markov chain of length $1.4 \times 10^{5}$, with a $1.4 \times 10^{4}$ burn-in time. The best orbital solution had a reduced $\chi^{2}$ of 1.0 
TABLE 7

Astrometric Solution for GJ 802AB

\begin{tabular}{|c|c|c|c|}
\hline Parameter & Unconstrained Fit & Fixed $M_{\mathrm{A}}$ & Constrained $M_{\mathrm{A}}$ \\
\hline 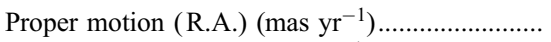 & $877.7 \pm 1.0$ & $877.7 \pm 1.0$ & $877.7 \pm 1.0$ \\
\hline 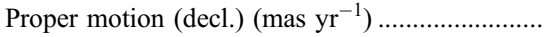 & $1722.11 \pm 0.34$ & $1722.2 \pm 1.0$ & $1722.1 \pm 1.0$ \\
\hline 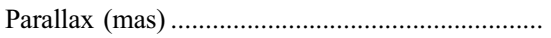 & $62.2 \pm 1.8$ & $63.9 \pm 1.3$ & $63.5 \pm 1.3$ \\
\hline 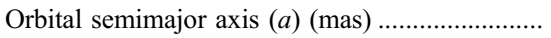 & $97.5 \pm 5.0$ & $92.4 \pm 2.0$ & $92.9 \pm 2.1$ \\
\hline Photocenter semimajor axis $(\alpha)$ (mas). & $17.4 \pm 1.2$ & $17.2 \pm 0.9$ & $17.2 \pm 1.0$ \\
\hline 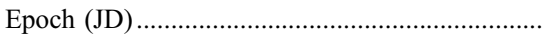 & $2,453,040 \pm 14$ & $2,453,028 \pm 15$ & $2,453,031 \pm 13$ \\
\hline Period (days) .................. & $1105 \pm 9$ & $1105 \pm 9$ & $1104 \pm 9$ \\
\hline Eccentricity ........................... & $0.40 \pm 0.08$ & $0.35 \pm 0.05$ & $0.35 \pm 0.05$ \\
\hline 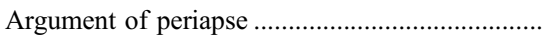 & $90.4 \pm 4.3$ & $89.0 \pm 5.5$ & $89.6 \pm 4.8$ \\
\hline Longitude of ascending node ....... & $21.8 \pm 1.4$ & $22.7 \pm 1.4$ & $22.0 \pm 1.3$ \\
\hline 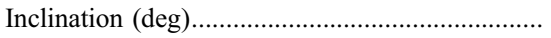 & $83.7 \pm 3.0$ & $81.1 \pm 3.1$ & $82.7 \pm 3.0$ \\
\hline Total mass $\left(M_{\odot}\right)$ & $0.426 \pm 0.078$ & $0.329 \pm 0.004$ & $0.343 \pm 0.012$ \\
\hline 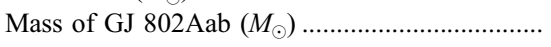 & $0.351 \pm 0.066$ & $0.268^{\mathrm{a}}$ & $0.280^{\mathrm{b}} \pm 0.010$ \\
\hline 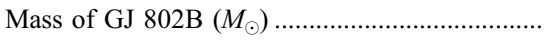 & $0.076 \pm 0.013$ & $0.061 \pm 0.004$ & $0.063 \pm 0.005$ \\
\hline
\end{tabular}

a Fixed from a mass $-K$ magnitude relationship (see text).

b Mass with error bars included as a priori information in the Monte Carlo fits.

because we chose the error in the STEPS data to be 1.7 mas in each axis. As in Pravdo et al. (2005) we used a $2 \pm 1$ mas conversion from relative to absolute parallax.

We first conducted an exploratory unconstrained fit to the data, but found that the errors on parallax and orbital semimajor axis were too large to give a useful total mass. This fit with single parameter $1 \sigma$ confidence limits is given in the first column of Table 7, and it has a mass for GJ 802Aab that is too high by $\sim 1.1 \sigma$ from the mass derived from the absolute magnitude of GJ 802Aab.

Therefore, we fixed the masses of GJ 802Aa and GJ 802Ab to be $0.134 M_{\odot}$, based on their absolute $K$ magnitudes and the models of Baraffe et al. (1998) (see $\S 3$ ). Note that the same value of parallax of 63 mas was used in both $\S 3$ and here in calculating the $K$-band absolute magnitude. This fit is given in the central column of Table 7 .

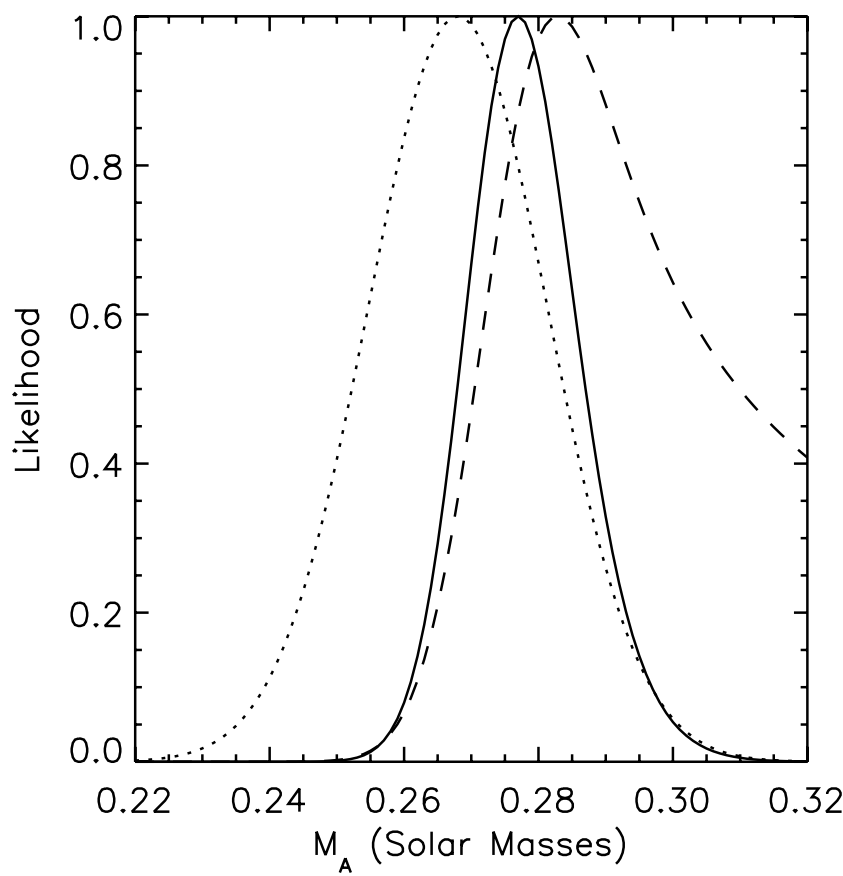

FIG. 4.-A priori constraint on the mass of GJ 802A from the $K$-band luminosity (dotted line), the radial velocity orbit (dashed line), and the joint likelihood (solid line).
Finally, we note that we cannot really "fix" the mass of GJ 802A as it has an uncertainty. We therefore calculated an a priori constraint on the mass of GJ 802Aab using the following: the mass $-K$ magnitude relationship with its assumed 5\% rms error (see $\S 3$ ); the value of $M \sin ^{3}(i)$ from Table 6 ; and an orbital orientation assumed random with $i<83^{\circ}$. Together these constraints give a $0.2775 \pm 0.0082 M_{\odot}$ for the mass of GJ 802Aab. The assumed likelihood curve resulting from these two constraints is given in Figure 4: it can be seen that a Gaussian likelihood curve is a good approximation for our prior knowledge of $M_{\mathrm{A}}$. The knowledge of this mass was added to the Markov chain Monte Carlo as an a priori constraint to give our preferred values, which appear in the final column of Table 8 . The final GJ 802B mass of $0.063 \pm 0.005 M_{\odot}$ is the most accurate mid to late L-dwarf mass reported in the literature so far.

Although the Markov chain Monte Carlo technique is excellent for accurately calculating posterior probability distributions of derived parameters, it can be difficult to intuitively understand the magnitude of derived uncertainties from the chain output. Therefore, we examine the origin of the $8 \%$ uncertainty on the GJ $802 \mathrm{~B}$ mass individually. The mass of the $\mathrm{B}$ component directly relates to the mass of the A components by the ratio of astrometric and orbital semimajor axes $a$ and $\alpha$ (e.g., McCarthy et al. 1988):

$$
M_{\mathrm{B}}=\frac{\alpha / a}{1-\alpha / a} M_{\mathrm{A}} \text {. }
$$

Therefore, a $4 \%$ uncertainty on the mass $M_{\mathrm{A}}$, a $6 \%$ uncertainty on $\alpha$ and a $2 \%$ uncertainty on $a$ combine to give a $8 \%$ uncertainty on $M_{\mathrm{B}}$. Although in the constrained fit the value and uncertainty for $a$ is influenced by the $M_{\mathrm{A}}$ constraint, this argument for the value

TABLE 8

Absolute Photometry for GJ 802

\begin{tabular}{ccccc}
\hline \hline Band & $m_{\mathrm{A}}$ & $m_{\mathrm{B}}$ & $M_{\mathrm{Aa}}\left(=M_{\mathrm{Ab}}\right)$ & $M_{\mathrm{B}}$ \\
\hline$V^{\mathrm{a}} \ldots \ldots \ldots .$. & 14.67 & $\ldots$ & 14.47 & $\ldots$ \\
$J_{\ldots \ldots \ldots \ldots .}$ & $9.57 \pm 0.02$ & $14.75 \pm 0.27$ & $9.34 \pm 0.05$ & $13.74 \pm 0.28$ \\
$H \ldots \ldots \ldots \ldots$. & $9.07 \pm 0.02$ & $14.13 \pm 0.09$ & $8.83 \pm 0.05$ & $13.14 \pm 0.10$ \\
$K \ldots \ldots \ldots \ldots$. & $8.76 \pm 0.01$ & $13.61 \pm 0.08$ & $8.53 \pm 0.05$ & $12.62 \pm 0.08$
\end{tabular}

${ }^{\text {a }}$ GJ $802 \mathrm{~B}$ is assumed to make a negligible contribution to $V$ band. 


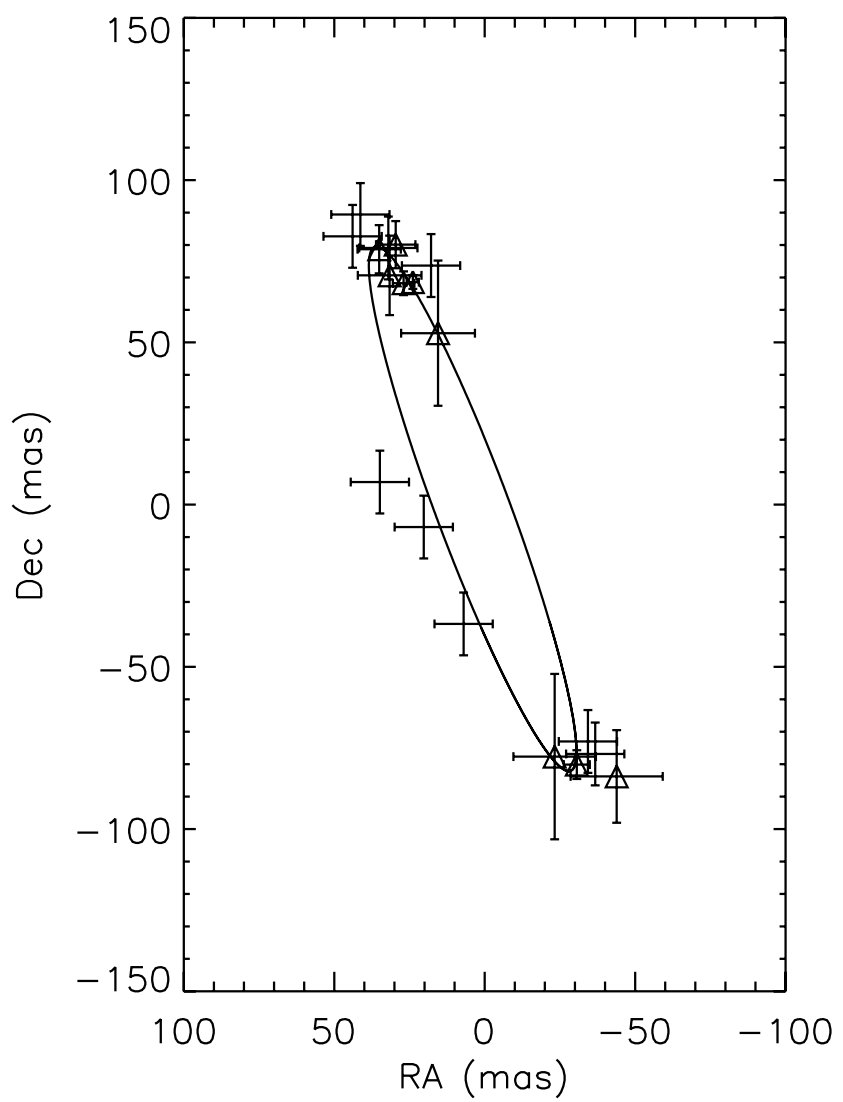

FIG. 5.-Best-fit orbit for GJ 802AB (oval), with aperture masking points (triangles) and scaled (see text) seeing-limited astrometry overplotted. [See the electronic edition of the Journal for a color version of this figure.]

and uncertainty of $M_{\mathrm{B}}$ changes little if the values for $a$ and $\alpha$ from the unconstrained fit are used.

The apparent photometry corresponding to this preferred fit is given in Table 8 . The apparent photometry for the system's combined light comes from Simbad and 2MASS (Cutri et al. 2003). The derived absolute photometry corresponding to individual components of the system (apportioning equal flux to the two components $\mathrm{Aa}$ and $\mathrm{Ab}$ ) is also given in this table. This photometry corresponds to a spectral type of L5-L7 for GJ 802B (Knapp et al. 2004).

The orbital fit for GJ 802AB is shown in Figure 5, with the photocenter astrometry measurements scaled by the ratio of total mass to GJ $802 \mathrm{~B}$ mass, and with parallax and proper motion removed so that all points can be plotted at the same scale. This plot also aids in developing an intuitive feel for our quoted mass error. The error in the ratio between the mass of GJ 802B and the total mass is the ratio of the photocenter and orbital semimajor axes (similar to eq. [2]), which we claim has a $7 \%$ error.

\section{COMPARISON WITH MODELS}

As discussed in Pravdo et al. (2005) the activity of GJ 802 (e.g., Fig. 1), implies that the system is $\gtrsim 6$ Gyr old. The kinematics of GJ 802 also imply that the system is old. The total proper motion of nearly $2^{\prime \prime} \mathrm{yr}^{-1}$ is 30 times the parallax, meaning that the tangential velocity of GJ 802 is $\sim 30$ AU per year, which is $\sim 140 \mathrm{~km} \mathrm{~s}^{-1}$. Based on the astrometric fit reported in Table 7 and the mean radial velocity from Tables 3 and 4, we have calculated the space motion of GJ 802 using the gal_uvw routine from the IDL Astronomy User's Library. ${ }^{1}$ This routine is in turn based on the mathematics in Johnson \& Soderblom (1987). The $(U, V, W)$ space motion calculated to be $(140,-51,20) \mathrm{km} \mathrm{s}^{-1}$, which is $(131,-39$, 27) $\mathrm{km} \mathrm{s}^{-1}$ with respect to the local standard of rest. The lag in $V$ and very high $U$ space velocity is quite inconsistent with the thindisk population. For example, the Hipparcos sample of nearby early $\mathrm{M}$ stars has a $U$ dispersion of $32 \mathrm{~km} \mathrm{~s}^{-1}$, and the sample earlier than spectral-type F5, representative of a $\sim 1$ Gyr old population, has a $U$ dispersion of only $22 \mathrm{~km} \mathrm{~s}^{-1}$ (Mignard 2000). The $39 \mathrm{~km} \mathrm{~s}^{-1} V$ lag of GJ 802 is unusually small for a Population II halo star, but is consistent with the thick disk (Casertano et al. 1990). GJ 802 is very unlikely to be a runaway star that is confused with the thick disk population, because any dynamical interaction capable of giving it $\mathrm{a} \sim 100 \mathrm{~km} \mathrm{~s}^{-1}$ peculiar velocity would also break the wide binary GJ $802 \mathrm{AB}$ apart. Therefore, these kinematic properties place GJ 802 at an age of $\sim 10 \mathrm{Gyr}$, and almost certainly older than 3 Gyr (Bensby et al. 2003).

We can compare these ages to the ages derived from the modeling of GJ 802B. Figure 6 shows the absolute magnitudes of GJ 802B compared to the DUSTY models of Baraffe et al. (2002). The colors of these models are clearly too red, a well-known property of these models for objects that are of mid-L or later spectral type (Baraffe et al. 2003). The predicted age is between 1 and $5 \mathrm{Gyr}$ in all bands. We chose not to plot the results from the COND models from the same group (Baraffe et al. 2003) because these models are much too blue and really only applicable to $\mathrm{T}$ dwarfs.

\footnotetext{
1 Available athttp://idlastro.gsfc.nasa.gov.
}
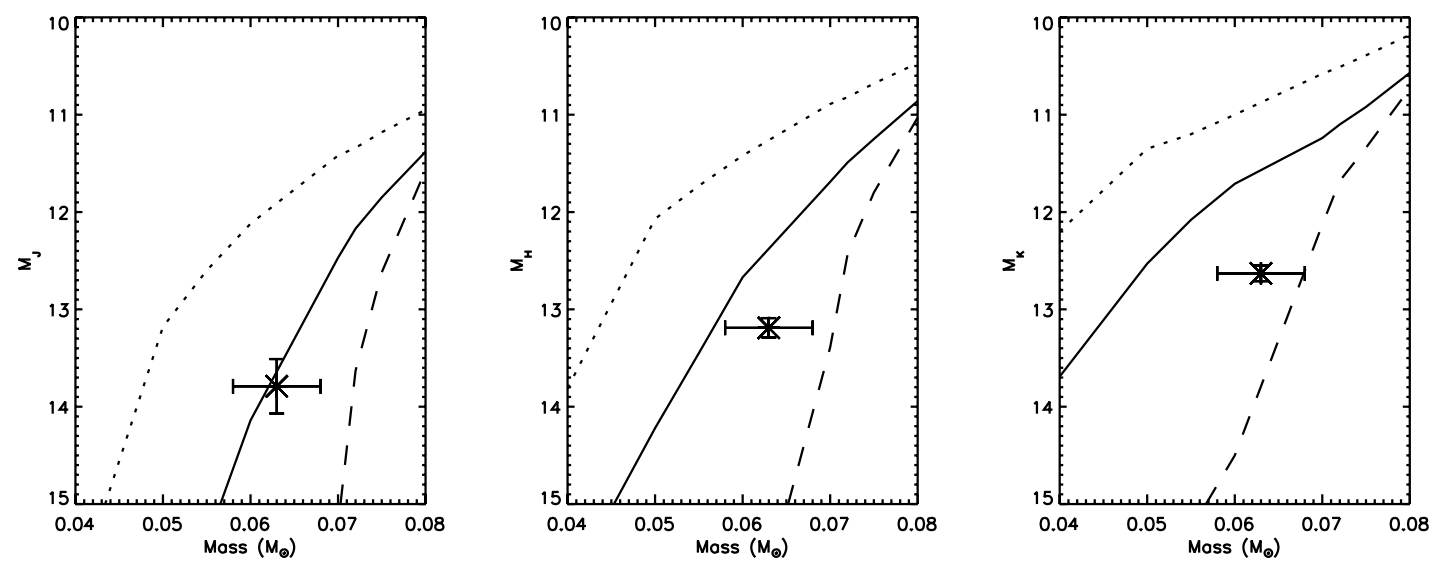

FIG. 6.- Absolute magnitudes of GJ 802B compared to the DUSTY models at $0.5,1$ and 5 Gyr. The comparison is for $J$ band (left), $H$ band (center), and $K$ band (right). Model ages are 0.5 Gyr (dotted line), 1 Gyr (solid line), and 5 Gyr (dashed line). 
Although these models clearly do not represent GJ 802B, mostly due to difficulties in opacity treatment and dust in brown dwarf atmospheres, it is expected that luminosity and effective temperature are a much more certain prediction of brown dwarf evolutionary models than fluxes in specific bands (Burrows \& Liebert 1993; Chabrier et al. 2000). A better comparison for the GJ 802B photometry is therefore possible with the models of Burrows et al. (2006), which correctly model the basic colors of L dwarfs and the $\mathrm{L}$ to $\mathrm{T}$ transition.

At $\log (g)=5.2$ (appropriate for a $\sim 0.06 M_{\odot}$ brown dwarf), the best-fitting model from Burrows et al. (2006) has absolute magnitudes $M_{J}=14.05, M_{H}=13.10$, and $M_{K}=12.78$. This model has $T_{\text {eff }}=1400 \mathrm{~K}$, with a total luminosity of $2.9 \times 10^{-5} L_{\odot}$. This model luminosity and temperature can now be compared with evolutionary models, placing a $0.063 M_{\odot}$ GJ $802 \mathrm{~B}$ at 1.9 Gyr according to the Burrows et al. (1997) track. GJ 802B would only be consistent with an old (i.e., $>5 \mathrm{Gyr}$ ) object if its mass were $0.07 M_{\odot}$, which is inconsistent with our dynamical mass at $1.5 \sigma$.

\section{CONCLUSIONS AND DISCUSSION}

GJ 802 is a triple system with component masses of $\sim 0.14$, 0.14 , and $0.063 M_{\odot}$. The inner pair of equal-mass stars, GJ 802Aab, has an orbital period of 0.795 days and an inclination between $74^{\circ}$ and $83^{\circ}$. The outer component, GJ $802 \mathrm{~B}$, has a $\sim 3$ yr orbit and a mass of $0.063 \pm 0.005 M_{\odot}$. The inclination of the GJ 802B orbit, $\sim 83^{\circ}$, is consistent with coplanarity. The most promising way to decrease the mass uncertainty would be to obtain an accurate radial velocity curve of the $3 \mathrm{yr}$ orbit, with an expected amplitude of $\sim 3 \mathrm{~km} \mathrm{~s}^{-1}$.

The brown dwarf "desert" is generally defined as a lack of low-mass companions around solar-type stars, but a similar lack of very unequal mass-ratio companions has been found around very low mass stars (Close et al. 2003). However, as a triple system, GJ 802 no longer fits in this category. In fact, there are a large number of brown dwarfs known in triple or higher order multiple systems. This entirely consistent with dynamical star formation simulations (Delgado-Donate et al. 2004), but may also relate to the details of the fragmentation process as a cloud with relatively high angular momentum collapses. Measuring the mutual inclination (i.e., degree of coplanarity) of this and other low-mass triples with long-baseline interferometry could help to determine the mechanism responsible for producing triples such as GJ 802.

The kinematics of GJ 802B place it in the thick disk, at an age of $\sim 10 \mathrm{Gyr}$, while the model-derived age for GJ $802 \mathrm{~B}$ is $\sim 2 \mathrm{Gyr}$. Although this discrepancy is only significant at the $93 \%$ confidence level so far $(1.5 \sigma)$, we list several possibilities for reconciling the discrepancy: (1) GJ 802Aab could actually have a total mass of $>0.30 M_{\odot}$, consistent with the astrometry, but be underluminous by $\sim 30 \%$ in $K$-band compared to field stars. GJ 802 B would then be placed right on the substellar boundary at $\sim 0.07 M_{\odot}$, a naively unlikely position given the very small sample of astrometric STEPS binaries from which GJ 802 was taken (Pravdo et al. 2005). (2) GJ 802Aab could have an apparent total mass of $>0.30 M_{\odot}$ because of an additional low-mass component in a $\sim 30$ day, just stable orbit. This could also increase our dynamical mass of GJ 802B to $\sim 0.07 M_{\odot}$. (3) GJ 802 could actually be $<2$ Gyr old but have experienced a unique dynamical past that gave it a high space velocity without tearing the wide binary apart. Or (4) models for old brown dwarfs are systematically underpredicting luminosities. These model errors could relate to, e.g., the effects of magnetic fields that can hinder heat flow in brown dwarfs (Chabrier et al. 2007). Given that GJ 802B is currently the only brown dwarf roughly a gigayear old or older with an accurate dynamical mass, we find this fourth possibility most likely — a hypothesis that will be testable within the next few years as more field brown dwarfs have accurate mass determinations.

M. J. I. would like to acknowledge Michelson Fellowship support from the Michelson Science Center and the NASA Navigator Program. A. L. K. is supported by a NASA Origins grant to L. Hillenbrand. This work is partially supported by the National Science Foundation under grants AST 05-06588 and AST 0705085 . Some of the data presented herein were obtained at the W. M. Keck Observatory, which is operated as a scientific partnership among the California Institute of Technology, the University of California and the National Aeronautics and Space Administration. The Observatory was made possible by the generous financial support of the W. M. Keck Foundation. The authors wish to recognize and acknowledge the very significant cultural role and reverence that the summit of Mauna Kea has always had within the indigenous Hawaiian community. We are most fortunate to have the opportunity to conduct observations from this mountain. Based partly on observations obtained at the Hale Telescope, Palomar Observatory, as part of a collaborative agreement between the California Institute of Technology, its divisions Caltech Optical Observatories and the Jet Propulsion Laboratory (operated for NASA), and Cornell University. Based partly on observations obtained at the Palomar 60 inch robotic telescope.

Facilities: Keck:II (NIRC2), Hale (PHARO,EAE), PO:1.5m

\section{REFERENCES}

Baldwin, J. E., Haniff, C. A., Mackay, C. D., \& Warner, P. J. 1986, Nature, 320, 595 Baraffe, I., Chabrier, G., Allard, F., \& Hauschildt, P. H. 1998, A\&A, 337, 403 2002, A\&A, 382, 563

Baraffe, I., Chabrier, G., Barman, T. S., Allard, F., \& Hauschildt, P. H. 2003, A\&A, 402, 701

Bensby, T., Feltzing, S., \& Lundström, I. 2003, A\&A, 410, 527

Brémaud, P. 1999, Markov Chains: Gibbs Fields, Monte Carlo Simulation, and Queues (New York: Springer), 290

Burrows, A., Hubbard, W. B., Lunine, J. I., \& Liebert, J. 2001, Rev. Mod. Phys., 73, 719

Burrows, A., \& Liebert, J. 1993, Rev. Mod. Phys., 65, 301

Burrows, A., Sudarsky, D., \& Hubeny, I. 2006, ApJ, 640, 1063

Burrows, A., et al. 1997, ApJ, 491, 856

Casertano, S., Ratnatunga, K. U., \& Bahcall, J. N. 1990, ApJ, 357, 435

Chabrier, G., Baraffe, I., Allard, F., \& Hauschildt, P. 2000, ApJ, 542, 464

Chabrier, G., Gallardo, J., \& Baraffe, I. 2007, A\&A, 472, L17

Close, L. M., Siegler, N., Freed, M., \& Biller, B. 2003, ApJ, 587, 407

Cutri, R. M., et al. 2003, The IRSA 2MASS All-Sky Point Source Catalog

(Pasadena: IPAC), http://irsa.ipac.caltech.edu/applications/Gator

Delfosse, X., et al. 2004, in ASP Conf. Ser. 318, Spectroscopically and Spatially Resolving the Components of the Close Binary Stars, ed. R. W. Hilditch, H. Hensberge, \& K. Pavlovski (San Francisco: ASP), 166

Delgado-Donate, E. J., Clarke, C. J., Bate, M. R., \& Hodgkin, S. T. 2004, MNRAS, 351, 617

Gizis, J. E., Reid, I. N., \& Hawley, S. L. 2002, AJ, 123, 3356

Hayward, T. L., Brandl, B., Pirger, B., Blacken, C., Gull, G. E., Schoenwald, J., \& Houck, J. R. 2001, PASP, 113, 105

Johnson, D. R. H., \& Soderblom, D. R. 1987, AJ, 93, 864

Knapp, G. R., et al. 2004, AJ, 127, 3553

Knox, L., Christensen, N., \& Skordis, C. 2001, ApJ, 563, L95

Lloyd, J. P., Martinache, F., Ireland, M. J., Monnier, J. D., Pravdo, S. H., Shaklan, S. B., \& Tuthill, P. G. 2006, ApJ, 650, L131

Luhman, K. L., Allers, K. N., Jaffe, D. T., Cushing, M. C., Williams, K. A., Slesnick, C. L., \& Vacca, W. D. 2007, ApJ, 659, 1629

McCarthy, Jr., D. W., Henry, T. J., Fleming, T. A., Saffer, R. A., Liebert, J., \& Christou, J. C. 1988, ApJ, 333, 943

Meyer, M. R., Edwards, S., Hinkle, K. H., \& Strom, S. E. 1998, ApJ, 508, 397

Michelson, A. A. 1920, ApJ, 51, 257 
Mignard, F. 2000, A\&A, 354, 522

Montalbán, J., D’Antona, F., \& Mazzitelli, I. 2000, A\&A, 360, 935

Naef, D., et al. 2003, A\&A, 410, 1051

Nidever, D. L., Marcy, G. W., Butler, R. P., Fischer, D. A., \& Vogt, S. S. 2002, ApJS, 141, 503

Pravdo, S. H., Shaklan, S. B., \& Lloyd, J. 2005, ApJ, 630, 528

Ségransan, D., Delfosse, X., Forveille, T., Beuzit, J.-L., Udry, S., Perrier, C., \& Mayor, M. 2000, A\&A, 364, 665
Stassun, K. G., Mathieu, R. D., \& Valenti, J. A. 2006, Nature, 440, 311 2007, ApJ, 664, 1154

Tuthill, P. G., Monnier, J. D., Danchi, W. C., Wishnow, E. H., \& Haniff, C. A. 2000, PASP, 112, 555

Zahn, J.-P., \& Bouchet, L. 1989, A\&A, 223, 112

Zapatero Osorio, M. R., Lane, B. F., Pavlenko, Y., Martín, E. L., Britton, M., \& Kulkarni, S. R. 2004, ApJ, 615, 958 